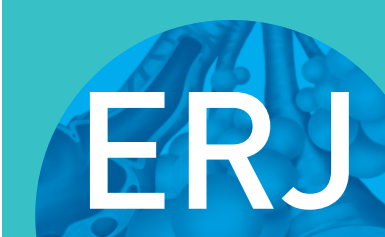

open research

\section{European Respiratory Society International Congress, Paris, 2018: highlights from the Clinical Assembly}

\author{
Lowie E.G.W. Vanfleteren (1) ${ }^{1}$, Iñigo Ojanguren ${ }^{2,3}$, Claire M. Nolan ${ }^{4}$, \\ Frits M.E. Franssen $\mathbb{1}^{5}$, Vasileios Andrianopoulos ${ }^{6}$, Aleksandar Grgic ${ }^{7}$, \\ Marlies van Dijk ${ }^{8}$, Dirk Jan Slebos ${ }^{8}$, Luke Daines ${ }^{9}$, Janwillem W.H. Kocks ${ }^{10,11}$ \\ and Nicolas Kahn ${ }^{12,13}$
}

Affiliations: ${ }^{1}$ COPD Center, Sahlgrenska University Hospital and Institute of Medicine, University of Gothenburg, Gothenburg, Sweden. ${ }^{2}$ Servicio de Neumología, Hospital Universitario Vall d'Hebron, Barcelona, Spain. ${ }^{3}$ Centro de Investigación en Red de Enfermedades Respiratorias (CIBERES), Instituto de Salud Carlos III (ISCIII), Barcelona, Spain. ${ }^{4}$ Harefield Pulmonary Rehabilitation and Muscle Research Laboratory, Royal Brompton and Harefield NHS Foundation Trust, London, UK. ${ }^{5}$ Dept of Research and Education, CIRO, Horn, The Netherlands. ${ }^{6}$ Institute for Pulmonary Rehabilitation Research, Schoen Klinik Berchtesgadener Land, Schoenau am Koenigssee, Germany. ${ }^{7}$ Dept of Nuclear Medicine, Saarland University Medical Center, Homburg, Germany. ${ }^{8}$ Dept of Pulmonary Diseases, University of Groningen, University Medical Center Groningen, Groningen, The Netherlands. ${ }^{9}$ Asthma UK Centre for Applied Research, Usher Institute of Population Health Sciences and Informatics, The University of Edinburgh, Edinburgh, UK. ${ }^{10}$ General Practitioners Research Institute, Groningen, The Netherlands. ${ }^{11}$ University of Groningen, University Medical Center Groningen, GRIAC Research Institute, Groningen, The Netherlands. ${ }^{12}$ Dept of Pneumology and Critical Care Medicine, Thoraxklinik, Heidelberg University Hospital, Heidelberg, Germany. ${ }^{13}$ Translational Lung Research Center Heidelberg, Member of the German Center for Lung Research, Heidelberg, Germany.

Correspondence: Lowie E.G.W. Vanfleteren, COPD Center, Sahlgrenska University Hospital and Institute of Medicine, University of Gothenburg, Vita Stråket 12, Gothenburg 413 45, Sweden.

E-mail: lowie.vanfleterenđavgregion.se

ABSTRACT This article contains highlights and a selection of the scientific advances from the European Respiratory Society's Clinical Assembly (Assembly 1 and its five respective groups) that were presented at the 2018 European Respiratory Society International Congress in Paris, France. The most relevant topics from each of the groups will be discussed, covering a wide range of areas including clinical problems, rehabilitation and chronic care, thoracic imaging, interventional pulmonology, and general practice and primary care. The newest research, actual data and highlight sessions will be discussed.

@ERSpublications

A highlights review of selected presentations from \#ERSCongress 2018 by the @ERStalk Clinical Assembly http://ow.ly/70Xu30mZ6t9

Cite this article as: Vanfleteren LEGW, Ojanguren I, Nolan CM, et al. European Respiratory Society International Congress, Paris, 2018: highlights from the Clinical Assembly. ERJ Open Res 2019; 5: 00176-2018 [https://doi.org/10.1183/23120541.00176-2018].

Received: Oct 032018 | Accepted after revision: Nov 132018

Copyright $\odot$ ERS 2019. This article is open access and distributed under the terms of the Creative Commons Attribution Non-Commercial Licence 4.0. 


\section{Introduction}

The European Respiratory Society (ERS) International Congress is the world's largest scientific and educational conference in the field of respiratory medicine. For the Paris 2018 Congress, 4317 abstracts were accepted for presentation. The Clinical Assembly of the ERS is the largest of the now 13 assemblies. In total, 970 abstracts were presented in 60 sessions related to this assembly and the five groups of which it consists. 26 clinical sessions were organised. It is impossible for any delegate to follow all scientific and clinical advances and breakthroughs presented during the Congress. This review aims to provide the esteemed reader with an overview of a few of the most interesting presentations of each group, as deemed noteworthy by the authors of this article.

\section{Group 1.1: Clinical problems}

\section{Session: Clinical year in review}

In this session, the best articles from diverse fields such as pulmonary rehabilitation and chronic care, asthma, chronic obstructive pulmonary disease (COPD), and interventional pneumology were reviewed by leading experts.

Martijn Spruit (the Netherlands) reviewed the year in rehabilitation and chronic care. He presented the "COMET study: a multicomponent home-based disease management programme versus routine care in severe COPD”, recently published in the European Respiratory Journal by KEssLER et al. [1]. The study was an international open-design clinical trial in COPD patients randomised 1:1 to the disease management intervention (a self-management programme, home telemonitoring, care coordination and medical management) or to the usual management practices at the study centre. The authors concluded that a novel multicomponent home-based COPD disease management intervention across four European countries did not achieve a statistically significant difference in the predefined primary outcome, although patients in the disease management group had 23\% fewer all-cause hospitalisation days than patients in the usual care control group. Afterwards, an already in-press randomised controlled trial (RCT) by Arbillaga-EtXarri et al. [2] entitled "Long-term efficacy and effectiveness of a behavioural and community-based exercise intervention (Urban Training) to increase physical activity in patients with COPD" was reviewed. The authors concluded that Urban Training, combining behavioural strategies with unsupervised outdoor walking, was efficacious in increasing physical activity after 12 months in COPD patients, with few safety concerns. Diverse characteristics such as the novel approach, the good design, the large sample size and the objectified physical activity of the study were emphasised during the presentation. Other new studies that compared a structured home-based rehabilitation programme [3] and Tai Chi [4] with conventional pulmonary rehabilitation in COPD were also analysed in this section.

The year in asthma was reviewed by Pascal Chanez (France), who addressed a large variety of recently published articles regarding mild and severe asthma. One of the selected articles with respect to mild asthma, "Inhaled combined budesonide-formoterol as needed in mild asthma" (SYGMA 1 trial) [5], investigated the superiority of as-needed budesonide-formoterol to as-needed terbutaline with regard to electronically recorded weeks with well-controlled asthma. In patients with mild asthma, as-needed budesonide-formoterol provided superior asthma symptom control to as-needed terbutaline, assessed according to electronically recorded weeks with well-controlled asthma, but was inferior to budesonide maintenance therapy. Along the same line, the SYGMA 2 trial entitled "As-needed budesonide-formoterol versus maintenance budesonide in mild asthma" [6] showed that in patients with mild asthma, budesonide-formoterol used as needed was noninferior to twice-daily budesonide with respect to the rate of severe asthma exacerbations during 52 weeks of treatment, but was inferior in controlling symptoms. Regarding severe asthma, two articles recently published in the New England Journal of Medicine related to dupilumab were highlighted: "Dupilumab efficacy and safety in moderate-to-severe uncontrolled asthma" [7] and "Efficacy and safety of dupilumab in glucocorticoid-dependent severe asthma" [8].

The year in COPD was reviewed by Wizia Wedzicha (UK), who presented a great cast of articles that approached many aspects of COPD such as physiopathology, follow-up and treatment. One of the first mentioned studies was a study by Agustí et al. [9] that was performed using data from the Framingham Offspring Cohort. The authors hypothesised that low lung function (forced expiratory volume in $1 \mathrm{~s}$ $(\mathrm{FEV} 1)<80 \%$ predicted) in early adulthood (25-40 years) was associated with higher prevalence and earlier incidence of respiratory, cardiovascular and metabolic abnormalities and premature death. They finally concluded that low peak lung function in early adulthood was a common finding, which could identify a group of individuals at risk of early comorbidities and premature death. Next, articles expanding on clinical aspects (trajectory after hospital admission [10] and exacerbations [11]) were then noted. In the last part of the section, articles regarding treatment therapies were analysed. Professor Wedzicha emphasised the large sample number of the patients included in the DYNAGITO trial [12], which had the objective of investigating the ability of tiotropium and olodaterol to prevent COPD exacerbations. She also 
highlighted the study entitled "Effect of lung deflation with indacaterol plus glycopyrronium on ventricular filling in patients with hyperinflation and COPD (CLAIM): a double-blind, randomised, crossover, placebo-controlled, single-centre trial" [13]. Studies investigating the benefits related to triple therapy in COPD patients were also presented $[14,15]$. The TRIBUTE study showed that among patients with symptomatic COPD, severe or very severe airflow limitation and an exacerbation history, extrafine beclometasone dipropionate, formoterol fumarate and glycopyrronium significantly reduced the rate of moderate-to-severe exacerbations compared with indacaterol-glycopyrronium, without increasing the risk of pneumonia [14]. Likewise, the IMPACT study revealed that triple therapy with fluticasone furoate, umeclidinium and vilanterol resulted in a lower rate of moderate or severe COPD exacerbations than fluticasone furoate-vilanterol or umeclidinium-vilanterol in COPD patients [15]. Finally, a trial addressing the utility of mepolizumab for eosinophilic COPD was reviewed [16]. PAVOrD et al. [16] reported that mepolizumab at a dose of $100 \mathrm{mg}$ was associated with a lower annual rate of moderate or severe exacerbations than placebo among patients with COPD and an eosinophilic phenotype.

Felix Herth (Germany) gave an overview of the year in interventional pulmonology. He stressed the relevance of progress in new data about thermoplasty. He presented the study entitled "Long-term outcomes of bronchial thermoplasty in subjects with severe asthma: a comparison of 3-year follow-up results from two prospective multicentre studies" (AIR2 and PAS2 trials) [17], which was published in the European Respiratory Journal in 2017. At year 3 after bronchial thermoplasty, the percentage of PAS2 subjects with severe exacerbations, emergency department visits and hospitalisations significantly decreased by $45 \%, 55 \%$ and $40 \%$, respectively, echoing the AIR2 results. In addition, the study entitled "Feasibility of bioengineered tracheal and bronchial reconstruction using stented aortic matrices" [18] was highlighted, among other studies.

Group 1.2: Pulmonary rehabilitation and chronic care

Session: Best abstracts in pulmonary rehabilitation and chronic care

The research presented in this oral presentation session included a variety of topics: post-hospitalisation pulmonary rehabilitation timing, exercise and pulmonary rehabilitation in asthma, skeletal muscle physiology, the prediction of mortality, and the pharmacological management of breathlessness.

LYNGBY KJAERGAARD et al. [19] undertook an RCT that investigated the differences in time to death and first hospitalisation at 1 year and other pulmonary rehabilitation outcomes between patients starting pulmonary rehabilitation within 2 weeks (early pulmonary rehabilitation) or 2 months (late pulmonary rehabilitation) of hospital discharge following a COPD exacerbation. There were no significant between-group differences in time to death or first hospitalisation, endurance capacity and quality of life. However, the early pulmonary rehabilitation group demonstrated greater improvements in exercise capacity, measured using the incremental shuttle walk test, at 2 months compared with late pulmonary rehabilitation (mean change $33.9 \mathrm{~m}, 95 \%$ CI 4.2-63.7 m), but this did not persist at 6 months.

Two studies investigated exercise training and pulmonary rehabilitation in asthma. First, an RCT compared a weight loss and exercise programme to a weight loss and sham programme (stretching exercises) in 55 obese asthmatic patients [20]. Compared with the control group, the intervention group demonstrated significantly greater improvements in light, moderate and vigorous physical activity levels, but not sedentary activity. Furthermore, significant between-group differences in frequency of asthma symptoms, depression as well as sleep efficiency and latency, favouring the weight loss and exercise programme, were observed. Second, Schultz et al. [21] undertook an RCT to explore the efficacy of a 3-week inpatient pulmonary rehabilitation programme compared with usual care in terms of asthma control and quality of life in 412 patients with uncontrolled asthma. Compared with usual care, the pulmonary rehabilitation group had statistically and clinically significantly better asthma control and quality of life on completion of pulmonary rehabilitation and at 3 months.

BleRvaQue et al. [22] compared skeletal muscle angiogenesis and capillary maturation of the vastus lateralis muscle in response to a 10 -week exercise programme between 14 COPD patients and seven matched healthy control subjects. The COPD patients were divided in two groups: group 1 trained at an intensity matched on the relative level $(n=7)$, whereas group 2 trained at an intensity matched on the absolute level of the training intensity of controls $(n=7)$. The control group achieved significantly greater improvements in quadriceps endurance than both COPD groups. Despite lower baseline capillary to fibre perimeter exchange index in the COPD groups, similar improvements in the capillary to fibre ratio were observed in all three groups. However, the capillary maturation index and the pro- to anti-angiogenic factor ratios were significantly lower in the COPD groups than the controls. The authors concluded that COPD patients have defective maturation during muscle angiogenesis in response to exercise training. 
Although pulmonary rehabilitation is effective in improving exercise capacity, considerable interpatient variability exists. In order to identify groups with differential exercise responses, KNEPPERs et al. [23] analysed quadriceps muscle molecular responses in 51 COPD patients following 4 weeks of inpatient pulmonary rehabilitation. Quadriceps muscle molecular markers were grouped by network-constrained clustering and their expression values were reduced to process scores that were used to allocate patients to a cluster. A number of clusters were identified, but only two differed according to pulmonary rehabilitation-induced autophagy, myogenesis, glucocorticoid signalling and oxidative metabolism regulation: clusters 0 and 1. There were no between-cluster differences in baseline characteristics, but following pulmonary rehabilitation a significantly higher proportion of cluster 0 patients exceeded the minimum clinically important difference in peak work load compared with cluster 1 patients $(61 \%$ versus $21 \%$; $\mathrm{p}=0.02)$.

There has been much interest in the utility of sit-to-stand tests in patients with chronic respiratory disease. JONES et al. [24] assessed the association between one of these tests, the five repetition sit-to-stand test (5STS), and all-cause mortality in 838 COPD patients. The cohort was followed for a mean of 1431 days and $20.3 \%$ died during this period. After adjusting for potential confounding variables, 5STS, expressed as a categorical and continuous variable, was independently associated with all-cause mortality (5STS-quartiles: HR 1.71, 95\% CI 1.05-2.78; 5STS-continuous: HR 1.01, 95\% CI 1.00-1.02). The authors concluded that 5STS may provide additional prognostic information in COPD assessments.

There is limited evidence to support the pharmacological management of chronic breathlessness. Accordingly, CuRRow et al. [25] undertook a double-blind placebo-controlled trial to measure the efficacy of extended-release oral morphine compared with placebo on breathlessness intensity in 284 opioid-naive, chronic breathlessness patients. Participants could take up to six doses of $2.5 \mathrm{mg}$ morphine (or placebo) daily for 7 days. There was no significant between-group difference in breathless intensity, measured using a $100-\mathrm{mm}$ visual analogue scale (VAS), following the intervention, but COPD patients with a modified Medical Research Council Dyspnoea score $\geqslant 3$ had significant reductions in "worst" breathlessness (mean change in VAS $-10.1 \mathrm{~mm}, 95 \% \mathrm{CI}-19.0--1.2 \mathrm{~mm}$ ).

\section{Group 1.3: Imaging}

Session: Imaging biomarkers and quantitative imaging

Advanced imaging techniques enable simultaneous assessment of pathological processes and physiological consequences by the acquisition of detailed anatomical and structural data [26]. Airway oedema and mucus hypersecretion may reduce the volume of visible airway lumens in imaging scanning [27]; however, it is not clear whether low airway visibility is associated with disease symptoms and impaired lung function in COPD patients. TANABE et al. [28] undertook a prospective study that examined whether the volume of visible airway lumens is associated with symptoms and lung function and can be changed in response to bronchodilation in COPD. They recruited 147 males with COPD and cross-sectionally examined computed tomography (CT) indexes, lung function and the COPD Assessment Test (CAT), while the effect of a long-acting anticholinergic bronchodilator was assessed in 37 males who underwent CT scans before and at 1 year after starting tiotropium therapy. Using sophisticated techniques, they identified a whole airway tree by segmenting the lumens and extracted portions of the tree in the right upper and middle-lower lobes (RUL and RMLL, respectively), and measured the RUL and RMLL volumes separately. Ratios of these volumes to the right lung volume (AWV\%) were used as airway visibility indexes. The investigators demonstrated that decreases in AWV\% for RUL and RMLL were significantly correlated with decreases in FEV1, and with increases in residual volume to total lung capacity ratio and CAT scores. They reported that AWV\% for RUL and RMLL was increased at 1-year follow-up, and that the changes in AWV\% were significantly greater in patients with substantial improvement in FEV1 compared with patients without. According to these findings, the airway visibility index reflects symptoms and lung function, and enables assessing regional bronchodilator responses in upper and middle-lower lobes in COPD.

Small airway disease (SAD) has been recognised as a central feature of COPD [29]. OsTRIDGE et al. [30] employed a novel quantitative CT (QCT) method (VIDA Lungprints; disease probability measure (DPM)) to assess the contribution of SAD in COPD. They recruited 38 mild-to-moderate COPD patients, 21 ex-smokers and 17 nonsmokers, who underwent chest CT in inspiration and expiration, and based on image analysis each voxel was classified as normal, emphysema or gas trapping due to SAD (DPMGT). Investigators demonstrated that DPMGT was significantly higher in COPD compared with ex-smokers or nonsmokers, and that there was an association between DPMGT and FEV1 and peripheral airway resistance, as well as strong associations between overall and lobar DPMGT values. There were also significant differences in DPMGT between lobes for all groups, being most marked in COPD. They reported that DPM analysis can detect SAD even in mild COPD and that this technique, by exhibiting strong associations with pulmonary functions and describing gas trapping distribution, may be a useful research tool in SAD. 
Airway remodelling is a well-established feature in severe refractory asthma (SRA) and COPD, along with persistent airflow limitation. The structural changes found on pathological examination of the remodelled airway wall have been shown to display similarities but also differences [31]. ОвоэКкі et al. [32] evaluated the intensity of remodelling of bronchi in SRA and COPD patients using QCT, and aimed at establishing a relationship between remodelling and airflow limitation. They recruited 10 SRA patients, 12 COPD patients and 11 healthy individuals with no respiratory or smoking history, who underwent chest CT and pulmonary function tests. Quantitative reconstruction of the CT data was performed, and wall thickness and lumen area were measured. Investigators reported that the results of QCT were intercorrelated with FEV1, forced vital capacity (FVC) and $\mathrm{FEV}_{1} / \mathrm{FVC} \%$, and with the occurrence of significant differences in wall thickness between SRA, COPD patients and healthy subjects. The wall thickness in generations 3-8 was significantly intensified in SRA compared with COPD and healthy groups, and there was a positive correlation between wall thickness (generations 5-9) and FEV1. They concluded that airway remodelling is more pronounced in SRA patients, and that there is a close relationship between airflow limitation and airway remodelling.

Segmental endotoxin challenge with lipopolysaccharide (LPS) can be used as a pharmacodynamic model to safely induce transient airway inflammation in the peripheral lung of healthy subjects and to test the anti-inflammatory effectiveness of new therapies by the use of bronchoalveolar lavage to quantify the LPS-induced cytokines [33]. ReNNE et al. [34] recruited 23 healthy smokers and induced LPS challenge into segments 4 or 5 of the right lung to quantify the oedema on turbo inversion recovery magnitude (TIRM) images and $\mathrm{T}_{1}$ maps (volume over threshold (VOT)) before contrast agent administration. They demonstrated that the percentage of neutrophilic cells was higher in LPS-challenged segments compared with baseline based on TIRM scores and VOT, and that the median contrast agent washout time remained stable but was different in the LPS-challenged segments compared with the saline segments (control) at $24 \mathrm{~h}$. The investigators reported that proton lung magnetic resonance imaging can detect regional oedema after segmental LPS challenge in healthy smokers and may be a promising tool for treatment monitoring in future trials.

\section{Group 1.4: Interventional pulmonology}

Session: Interventional pulmonology highlights

This oral presentation session focused on endoscopic interventions in obstructive lung diseases, with presentations about lung volume reduction treatment with endobronchial valves (EBVs), targeted lung denervation in COPD and bronchial thermoplasty in asthma.

12-month follow-up data of one-way valve treatment in patients with severe, heterogeneous emphysema and hyperinflation from two multicentre international RCTs were presented.

CRINER et al. [35] presented the results of the EMPROVE trial, in which 172 patients were 2:1 randomised to endoscopic lung volume reduction using the Spiration Valve System (Spiration, Center Valley, PA, USA) or to receive standard of care (SoC), using only CT fissure integrity $>90 \%$ as measurement for collateral ventilation. At 12 -month follow-up, $37.3 \%$ of patients had an increase in FEV1 of $>100 \mathrm{~mL}$ and an improvement of -9.5 points on the St George's Respiratory Questionnaire. Remarkably, there was no significant change in 6-min walk distance (6MWD). Pneumothorax as a serious adverse event (SAE) occurred in 12.4\%. Between 6 and 12 months there was no significant difference in SAEs between groups.

CRINER et al. [36] presented results of the LIBERATE trial, which investigated the Zephyr EBV (PulmonX, Redwood City, CA, USA) in 190 patients with a 2:1 randomisation to treatment with EBV or SoC, using the Chartis measurement of interlobar collateral ventilation to assess eligibility. At 12-month follow-up, $47.7 \%$ of patients had $\geqslant 15 \%$ increase of FEV1. There was an improvement in $6 \mathrm{MWD}(+39.31 \mathrm{~m})$ and quality of life (SGRQ -7.05 points). Pneumothorax as an SAE occurred in $26.6 \%$ in the first 45 days after treatment. Between 45 days to 12 months there was no significant difference in SAEs between groups.

When selecting patients for EBV treatment, absence of collateral ventilation between the treatment target and ipsilateral lobe is important for a successful outcome [37]. In a retrospective analysis, RuwwE-GLÖSENKAMP et al. [38] reported that fissure completeness scores (FCS), assessed by QCT analysis (StratX; PulmonX), were correlated with Chartis assessment of collateral ventilation. In general, there was a good correlation between Chartis and quantitative CT analysis. Nonetheless, in $13.8 \%$ of cases where the FCS was $>95 \%$, the Chartis measurement turned out collateral ventilation positive. Only when FCS was $<85.6 \%$ were there no Chartis measurements where the outcome was collateral ventilation negative.

Selecting the right target lobe for treatment with EBV can be challenging. TeE et al. [39] investigated the combination of quantitative analysis of a CT scan combined with ventilation/perfusion single photon emission CT (VQ-SPECT). This technique enables the share of each individual lobe to be determined for lung volume, perfusion and ventilation. Using this information they calculated the so-called Ventilation- 
FIGURE 1 Nuvaira dual-cooled lung denervation balloon catheter.

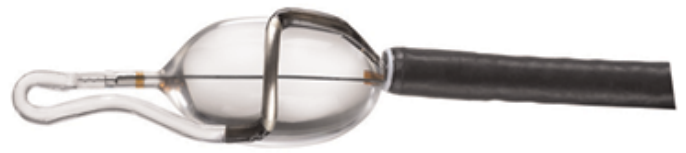

Perfusion Differentiation Index (VQDI) $=($ ventilation $(\%$ of total ventilation $) /$ volume (\% of total lung volume $) \times($ perfusion $(\%$ of total perfusion $) /$ volume ( $\%$ of total lung volume) $)$. A lobe with a low VQDI was proposed to be a good target lobe. When comparing VQDI to CT combined with a planar perfusion scan in 18 patients, the proposed target lobe was the same in $89 \%$ of cases. Furthermore, there was a significant reduction in VQDI in the target lobe 3 and 6 months after treatment, but notably there was no significant difference in VQDI for the other lobes.

Targeted lung denervation is a novel bronchoscopic treatment for patients with symptomatic COPD (Global Initiative for Chronic Obstructive Lung Disease B and D) where the intention is to achieve radiofrequency ablation of the parasympathetic nerves around the main bronchi using a special catheter (figure 1) [40]. SLebos et al. [41] presented data from a 1:1 randomised full sham-controlled double-blinded multicentre trial showing the feasibility and safety of this treatment using the Nuvaira Lung Denervation System (Nuvaira, Minneapolis, MN, USA) in patients with moderate-to-severe COPD. Active treatment showed significant reduction in respiratory events over sham controls (32\% versus $71 \%$; $\mathrm{p}=0.0008$ ). At up to 1 -year follow-up, there was also a $>50 \%$ decrease in severe COPD exacerbations for the targeted lung denervation group compared with sham controls.

Bronchial thermoplasty is a bronchoscopic treatment for patients with severe asthma where radiofrequency energy is delivered to the airways to reduce airway smooth muscle (ASM) mass. Bronchial thermoplasty has been shown to reduce the exacerbation rate and improve asthma-related quality of life [42]. However, the exact mechanism of action of bronchial thermoplasty remains uncertain and it is unclear which asthma phenotype responds best. D'Hooghe et al. [43] studied bronchial biopsies and spirometry at baseline and 6 months after bronchial thermoplasty in 16 patients. There was no change in FEV 1 after bronchial thermoplasty. There was, however, a significant negative relation between ASM mass decrease (quantified using desmin immunostaining) and baseline $\mathrm{FEV}$, leading the authors to conclude that patients with a lower FEV1 show the most reduction of ASM after bronchial thermoplasty. Data on clinical improvements were not shown.

Optical coherence tomography (OCT) is an imaging technique with near-infrared light used to obtain cross-sectional images of the airway which can be performed during bronchoscopy. Goorsenberg et al. [44] presented the results of a study in 15 patients with severe asthma in which OCT was performed directly following and 6 weeks after bronchial thermoplasty. Three OCT patterns where identified: bronchial oedema, peribronchial oedema and epithelial sloughing. The majority of airways showed these three patterns right after bronchial thermoplasty, with a reduction in incidence and intensity at the 6-week follow-up. Notably, the effect of treatment extended beyond the ASM layer and distally from the treated area. It is not yet clear whether these findings have a correlation with treatment response.

Finally, LANGTON et al. [45] studied patients with severe asthma who underwent bronchial thermoplasty, divided into two groups based on reversibility of airflow obstruction ( $\mathrm{n}=18$ reversible and $\mathrm{n}=24$ irreversible). At the 6-month follow-up there was a significant reduction in symptoms, exacerbation rate, use of reliever medication and need for maintenance oral corticosteroids in the overall group. There were, however, no significant differences between both groups, leading the authors to conclude that reversibility in airflow obstruction does not influence response to bronchial thermoplasty.

\section{Group 1.5: General practice and primary care}

The value of primary healthcare in improving respiratory outcomes was recognised throughout the 2018 ERS Congress. In the Global Initiative for Asthma session, Eric Bateman (South Africa) and Helen Reddel (Australia) both highlighted the need for better implementation of guidelines in primary care as a key 
determinant in improving global asthma outcomes. It is fitting then that the primary care day programme offered a new look at providing healthcare for long-term conditions.

David Darmon (France) began the primary care day by sharing his experience of telemedicine and routinely collected data as opportunities to enhance community-based respiratory care. Although enthusiastic, he cautioned that telemedicine should augment existing practices and relationships between primary and secondary care. Patient ambassador Isabel Saraiva (Portugal) also highlighted the positive impact of technology on patient experience. For instance, the European Lung Foundation has accessible, authoritative information for patients, and successfully uses wide-ranging media to increase awareness of respiratory disease and causes such as smoking and air pollution. Evidence linking airborne pollutants and allergen exposure to respiratory disease was later described by Jeroen Douwes (New Zealand), who shared results demonstrating the protective effects of green spaces on asthma development [46].

Progressing from disease determinants to clinical diagnosis, Luke Daines (UK) focused on asthma diagnosis and summarised concerns and reasons for asthma misdiagnosis [47, 48]. Until asthma phenotypes are operationalised, increasing the availability and quality of diagnostic tests in primary care is important.

The remaining talks gave new perspectives on disease management. Providing instructions for individuals with asthma in the event of worsening symptoms is key in supporting asthma self-management [49]. Tim Harrison (UK) reported pragmatic RCT results demonstrating that adults and adolescents who used a personalised asthma action plan, recommending a temporary quadrupling of inhaled corticosteroids when asthma control deteriorated, had fewer severe asthma exacerbations than individuals who did not increase the dose [50]. Interestingly, JACKSON et al. [51] investigated a similar question in children, but found no benefit from quintupling inhaled steroids. That different outcomes were observed between children and adults may not be surprising. However, adherence may be an additional factor. The RCT in children required at least $75 \%$ participant adherence to maintenance therapy [51]. The study in adults did not require a minimum adherence level [50]. Perhaps by instructing inhaled corticosteroids to be quadrupled, adults who had been nonadherent benefitted from re-establishing a therapeutic treatment dose.

Building on these observations, Janwillem Kocks (the Netherlands) recommended ways to optimise inhaled therapies by addressing nonadherence, inhaler choice and education. Responding to a vote, $44 \%$ of the audience acknowledged they spent more time prescribing an inhaler than demonstrating how to use it. New educational strategies were proposed, and the ability to give direct feedback on adherence and technique using smart inhalers was suggested as an opportunity for the near future.

Finally, Mike Thomas (UK) shared practical strategies for relieving breathlessness. Individuals with asthma may have impaired quality of life despite pharmacotherapy. The BREATHE trial demonstrated that compared with usual care, breathing retraining improved quality of life in those with incompletely controlled asthma [52]. The intervention was effective when individuals completed sessions by themselves. Therefore, with resources freely available (www.breathetrain.co.uk), clinicians have a greater breadth of treatments to offer.

Sharing new perspectives on respiratory healthcare prompted much discussion at the primary care day. The opportunities to meet and share ideas with delegates with different expertise from across the world was a huge advantage of the ERS Congress in Paris, and one that we very much look forward to again at Madrid 2019.

\section{Concluding remarks}

The authors of this article hope that this short summary of the impressive amount of lung research and advances in pulmonary care presented through the Clinical Assembly of the ERS creates curiosity to follow-up on topics of interest to each individual reader.

It was our goal to stimulate discussion and exchange of scientific novelties and clinical developments. We also hope to have encouraged the readership to attend the 2019 ERS International Congress in Madrid (https://erscongress.org).

Conflict of interest: L.E.G.W. Vanfleteren reports receiving personal fees from AstraZeneca, Chiesi, PulmonX, GSK and Novartis, outside the submitted work. I. Ojanguren reports receiving grants from Mundipharma, speaker's fees from Boehringer Ingelheim, AstraZeneca, Teva and Novartis, and consulting fees from GSK, outside the submitted work. C.M. Nolan reports that she is co-author on one of the abstracts presented in this article. F.M.E. Franssen reports receiving personal fees from AstraZeneca, Chiesi, Boehringer Ingelheim, GSK, Teva and Novartis, outside the submitted work. V. Andrianopoulos has nothing to disclose A. Grgic has nothing to disclose. M. van Dijk has nothing to disclose. D.J. Slebos reports receiving reimbursement, devices for treatments for clinical trial trials performed at his institution (NCT01101958, NCT01796392 and NCT02025205), reimbursement for travel expenses, and speakers' fees for presentations at scientific and educational meetings from PulmonX Inc. (USA); reimbursement for clinical trial trials performed in his institution (NCT02058459 and NCT01483534), devices for treatments, reimbursement for travel 
expenses, and speakers' fees for presentations at scientific and educational meetings from Nuvaira Inc. (USA); reimbursement for clinical trial trials performed in his institution, devices for treatments, reimbursement for travel expenses and speakers' fees for presentations at scientific and educational meetings from, and has acted as a physician advisor to CSA Medical (USA); reimbursement for clinical trial trials performed in his institution (NCT02059057, NCT02179125, NCT01608490, NCT01421082, NCT01328899 and NCT01220908), devices for treatments, reimbursement for travel expenses, speakers' fees for presentations at scientific and educational meetings from, and has acted as a physician advisor to PneumRx/BTG (USA/UK), outside the submitted work. He is co-author on one of the abstracts presented in this article. L. Daines has nothing to disclose. J.W.H. Kocks reports receiving grants and personal fees from AstraZeneca and Boehringer Ingelheim, personal fees and nonfinancial support from GSK, grants from Chiesi and Mundi Pharma, outside the submitted work. He is an author of one of the presentations mentioned in this article As Secretary of ERS Assembly 1, he was co-responsible in developing the symposium. N. Kahn has nothing to disclose.

Support statement: V. Andrianopoulos is the recipient of an ERS/EU RESPIRE2 Marie Sklodowska-Curie Postdoctoral Research Fellowship (MCF (8465)-2015).

\section{References}

1 Kessler R, Casan-Clara P, Koehler D, et al. COMET: a multicomponent home-based disease-management programme versus routine care in severe COPD. Eur Respir J 2018; 51: 1701612.

2 Arbillaga-Etxarri A, Gimeno-Santos E, Barberan-Garcia A, et al. Long-term efficacy and effectiveness of a behavioural and community-based exercise intervention (Urban Training) to increase physical activity in patients with COPD. A randomised controlled trial. Eur Respir J 2018: 52: 1800063.

3 Horton EJ, Mitchell KE, Johnson-Warrington V, et al. Comparison of a structured home-based rehabilitation programme with conventional supervised pulmonary rehabilitation: a randomised non-inferiority trial. Thorax 2018; 73: 29-36.

4 Polkey MI, Qiu ZH, Zhou L, et al. Tai Chi and pulmonary rehabilitation compared for treatment-naive patients with COPD: a randomized controlled trial. Chest 2018; 153: 1116-1124.

5 O’Byrne PM, FitzGerald JM, Bateman ED, et al. Inhaled combined budesonide-formoterol as needed in mild asthma. N Engl J Med 2018; 378: 1865-1876.

6 Bateman ED, Reddel HK, O’Byrne PM, et al. As-needed budesonide-formoterol versus maintenance budesonide in mild asthma. N Engl J Med 2018; 378: 1877-1887.

7 Castro M, Corren J, Pavord ID, et al. Dupilumab efficacy and safety in moderate-to-severe uncontrolled asthma. N Engl J Med 2018; 378: 2486-2496.

8 Rabe KF, Nair P, Brusselle G, et al. Efficacy and safety of dupilumab in glucocorticoid-dependent severe asthma. N Engl J Med 2018; 378: 2475-2485.

9 Agustí A, Noell G, Brugada J, et al. Lung function in early adulthood and health in later life: a transgenerational cohort analysis. Lancet Respir Med 2017; 5: 935-945.

10 Lindenauer PK, Dharmarajan K, Qin L, et al. Risk trajectories of readmission and death in the first year after hospitalization for chronic obstructive pulmonary disease. Am J Respir Crit Care Med 2018; 197: 1009-1017.

11 Kunisaki KM, Dransfield MT, Anderson JA, et al. Exacerbations of chronic obstructive pulmonary disease and cardiac events. A post hoc cohort analysis from the SUMMIT randomized clinical trial. Am J Respir Crit Care Med 2018; 198: 51-57.

12 Calverley PMA, Anzueto AR, Carter K, et al. Tiotropium and olodaterol in the prevention of chronic obstructive pulmonary disease exacerbations (DYNAGITO): a double-blind, randomised, parallel-group, active-controlled trial. Lancet Respir Med 2018; 6: 337-344.

13 Hohlfeld JM, Vogel-Claussen J, Biller H, et al. Effect of lung deflation with indacaterol plus glycopyrronium on ventricular filling in patients with hyperinflation and COPD (CLAIM): a double-blind, randomised, crossover, placebo-controlled, single-centre trial. Lancet Respir Med 2018; 6: 368-378.

14 Papi A, Vestbo J, Fabbri L, et al. Extrafine inhaled triple therapy versus dual bronchodilator therapy in chronic obstructive pulmonary disease (TRIBUTE): a double-blind, parallel group, randomised controlled trial. Lancet 2018; 391: 1076-1084.

15 Lipson DA, Barnhart F, Brealey N, et al. Once-daily single-inhaler triple versus dual therapy in patients with COPD. N Engl J Med 2018; 378: 1671-1680.

16 Pavord ID, Chanez P, Criner GJ, et al. Mepolizumab for eosinophilic chronic obstructive pulmonary disease N Engl J Med 2017; 377: 1613-1629.

17 Chupp G, Laviolette M, Cohn L, et al. Long-term outcomes of bronchial thermoplasty in subjects with severe asthma: a comparison of 3-year follow-up results from two prospective multicentre studies. Eur Respir J 2017; 50: 1700017.

18 Martinod E, Chouahnia K, Radu DM, et al. Feasibility of bioengineered tracheal and bronchial reconstruction using stented aortic matrices. JAMA 2018; 319: 2212-2222.

19 Lyngby Kjaergaard J, Juhl C, Rosenberg M, et al. Early pulmonary rehabilitation after acute exacerbation of COPD. Eur Respir J 2018; 52: Suppl. 62, OA1618.

20 Freitas P, Silva A, Ferreira P, et al. Exercise improves physical activity and comorbidities in obese adults with asthma. Eur Respir J 2018; 52: Suppl. 62, OA1619.

21 Schultz K, Wittmann M, Wagner R, et al. Effectiveness of pulmonary rehabilitation for patients with asthma: EPRA-RCT. Eur Respir J 2018; 52: Suppl. 62, OA1620.

22 Blervaque L, Pomiès $\mathrm{P}$, Catteau $\mathrm{M}$, et al. Pericyte coverage of skeletal muscle capillaries is impaired during training-induced angiogenesis in COPD patients. Eur Respir J 2018; 52: Suppl. 62, OA1621.

23 Kneppers A, Haast R, Langen R, et al. Unbiased analysis of skeletal muscle molecular responses upon pulmonary rehabilitation in advanced COPD. Eur Respir J 2018; 52: Suppl. 62, OA1622.

24 Jones S, Nolan C, Patel S, et al. Five-repetition sit-to-stand and mortality in COPD: a prospective cohort study. Eur Respir J 2018; 52: Suppl. 62, OA1623.

25 Currow D, Louw S, McCloud P, et al. Regular extended release morphine for chronic breathlessness: a multi-centre double-blind RCT. Eur Respir J 2018; 52: Suppl. 62, OA1624.

26 Milne S, King GG. Advanced imaging in COPD: insights into pulmonary pathophysiology. J Thorac Dis 2014; 6 : 1570-1585. 

asthma. J Thorac Imaging 2011; 26: 290-300.

lung function in COPD. Eur Respir J 2018; 52: Suppl. 62, OA3794

29 Koo HK, Vasilescu DM, Booth S, et al. Small airways disease in mild and moderate chronic obstructive pulmonary disease: a cross-sectional study. Lancet Respir Med 2018; 6: 591-602.

30 Ostridge K, Gove K, Kirby M, et al. Using DPM CT analysis to assess the contribution of small airways disease in COPD. Eur Respir J 2018; 52: Suppl. 62, OA3795.

31 Dournes G, Laurent F. Airway remodelling in asthma and COPD: findings, similarities, and differences using quantitative CT. Pulm Med 2012; 2012: 670414

32 Obojski A, Patyk M, Parkitna-Patyk M, et al. Quantitative computed tomography imaging in asthma and chronic obstructive pulmonary disease. Eur Respir J 2018; 52: Suppl. 62, OA3796.

33 Holz O, Tan L, Schaumann F, et al. Inter- and intrasubject variability of the inflammatory response to segmental endotoxin challenge in healthy volunteers. Pulm Pharmacol Ther 2015; 35: 50-59.

34 Renne J, Biller $\mathrm{H}$, Gutberlet $\mathrm{M}$, et al. Regional detection of edema following segmental LPS challenge using functional MR imaging. Eur Respir J 2018; 52: Suppl. 62, OA3798.

35 Criner GJ, Delage A, Voelker K. Endobronchial valves for severe emphysema - 12-month results of the EMPROVE trial. Eur Respir J 2018; 52: Suppl. 62, OA4928.

36 Criner GJ, Sue R, Wright S, et al. A multicenter RCT of Zephyr endobronchial valve treatment in heterogeneous emphysema (LIBERATE). Am J Respir Crit Care Med 2018; 198: 1151-1164.

37 Koster TD, van Rikxoort EM, Huebner RH, et al. Predicting lung volume reduction after endobronchial valve therapy is maximized using a combination of diagnostic tools. Respiration 2016; 92: 150-157.

38 Ruwwe-Glösenkamp C, Thelig D, Döllinger F, et al. Characterization of Chartis phenotypes based on fissure completeness scores. Eur Respir J 2018; 52: Suppl. 62, OA4931.

39 Tee V, Nguyen P, Grosser D, et al. Use of ventilation-perfusion single-photon emission computed tomography (VQ SPECT) to select the target lobe for endobronchial valves lung volume reduction (EBV-LVR). Eur Respir 2018; 52: Suppl. 62, OA4930.

40 Slebos DJ, Klooster K, Koegelenberg CF, et al. Targeted lung denervation for moderate to severe COPD: a pilot study. Thorax 2015; 70: 411-419.

41 Slebos D-J, Shah P, Herth F, et al. A double-blind, randomized, sham-controlled study of targeted lung denervation in patients with moderate to severe COPD. Eur Respir J 2018; 52: Suppl. 62, OA4929.

42 Castro M, Rubin AS, Laviolette M, et al. Effectiveness and safety of bronchial thermoplasty in the treatment of severe asthma: a multicenter, randomized, double-blind, sham-controlled clinical trial. Am J Respir Crit Care Med 2010; 181: 116-124

43 d'Hooghe JNS, Ten Hacken NHT, Weersink EJM, et al. Airway smooth muscle mass reduction after bronchial thermoplasty in asthmatics correlates with FEV1. Eur Respir J 2018; 52: Suppl. 62, OA4933.

44 Goorsenberg AWM, d'Hooghe JNS, De Bruin DM, et al. Acute airway effects of bronchial thermoplasty assessed by optical coherence tomography (OCT). Eur Respir J 2018; 52: Suppl. 62, OA4934

45 Langton D, Ing A, Fielding D, et al. Bronchodilator responsiveness as a success predictor for bronchial thermoplasty. Eur Respir J 2018; 52: Suppl. 62, OA4935.

46 Donovan GH, Gatziolis D, Longley I, et al. Vegetation diversity protects against childhood asthma: results from a large New Zealand birth cohort. Nat Plants 2018; 4: 358-364.

47 Aaron SD, Vandemheen KL, FitzGerald JM, et al. Reevaluation of diagnosis in adults with physician-diagnosed asthma. JAMA 2017; 317: 269-279.

48 Looijmans-van den Akker I, van Luijn K, Verheij T. Overdiagnosis of asthma in children in primary care: a retrospective analysis. Br J Gen Pract 2016; 66: e152-e157.

49 Gibson PG, Powell H. Written action plans for asthma: an evidence-based review of the key components. Thorax 2004; 59: 94-99.

50 McKeever T, Mortimer K, Wilson A, et al. Quadrupling inhaled glucocorticoid dose to abort asthma exacerbations. N Engl J Med 2018; 378: 902-910.

51 Jackson DJ, Bacharier LB, Mauger DT, et al. Quintupling inhaled glucocorticoids to prevent childhood asthma exacerbations. N Engl J Med 2018; 378: 891-901.

52 Bruton A, Lee A, Yardley L, et al. Physiotherapy breathing retraining for asthma: a randomised controlled trial. Lancet Respir Med 2018; 6: 19-28. 